BI-TP-2012-12

March 29, 2012

\title{
Resummation scheme for 3d Yang-Mills and the two-loop magnetic mass for hot gauge theories
}

\author{
Daniel Bieletzki ${ }^{1}$, Kilian Lessmeier ${ }^{2}$, Owe Philipsen ${ }^{1}$, York Schröder ${ }^{2}$ \\ ${ }^{1}$ Institut für Theoretische Physik, Goethe-Universität Frankfurt, \\ Max-von-Laue-Str. 1, 60438 Frankfurt am Main, Germany \\ 2 Fakultät für Physik, Universität Bielefeld, D-33501 Bielefeld, Germany
}

\begin{abstract}
Perturbation theory for non-Abelian gauge theories at finite temperature is plagued by infrared divergences caused by magnetic soft modes $\sim g^{2} T$, which correspond to the fields of a 3d Yang-Mills theory. We revisit a gauge invariant resummation scheme to solve this problem by self-consistent mass generation using an auxiliary scalar field, improving over previous attempts in two respects. First, we generalise earlier $\mathrm{SU}(2)$ treatments to $\mathrm{SU}(N)$. Second, we obtain a gauge independent two-loop gap equation, correcting an error in the literature. The resulting two-loop approximation to the magnetic mass represents a $\sim 15 \%$ correction to the leading one-loop value, indicating a reasonable convergence of the resummation.
\end{abstract}




\section{Introduction}

From heavy ion collision experiments to astroparticle physics and cosmology, there is a need for theoretical predictions from finite temperature gauge theories within and beyond the standard model. Since Monte Carlo simulations of lattice gauge theories do not work for finite baryon densities or dynamical problems involving real time, analytical approaches are warranted as well. Perturbation theory for equilibrium quantities of non-Abelian gauge theories at finite temperature $T$ features three scales: a hard scale $\pi T$ of the non-zero Matsubara modes related to the compactified Euclidean time direction, the soft and ultra-soft scales $g T, g^{2} T$, with gauge coupling $g$, which are associated with the screening of colour-electric and colour-magnetic gauge fields, respectively. Since the dimensionless expansion parameter for the latter features a mass (or momentum) scale in the denominator, $\sim g^{2} T / E(\mathbf{p})$, prohibitive infrared divergences occur in the perturbative series when bare, massless propagators are used. This is known as the Linde problem [1, 2]. Since the dynamically generated magnetic screening mass is itself $m \sim g^{2} T$, the problem cannot be cured to any finite order in ordinary perturbation theory. On the other hand, evidence from gauge fixed lattice simulations (see [3] and references therein) as well as gauge invariant simulations of field strength correlators [4] is consistent with an effectively massive gluon propagator. Moreover, a dynamically generated gauge boson mass plays a prominent role in a Hamiltonian description of three-dimensional Yang-Mills theory [5, 6] and is of renewed interest in the context of a Higgs-less electroweak gauge sector, see e.g. [7].

In this work we systematise a resummation method for 3d Yang-Mills theory, viz. the magnetic sector of finite $T$ gauge theories, which has been proposed some time ago 8]. The general idea is to screen the infrared divergences by adding a gauge invariant mass term, which gets subtracted again at higher order, resulting in a resummation of the loop expansion. The mass term is not unique and several possibilities have been tried at one-loop [9, 10, 11]. Under the name of screened perturbation theory similar techniques have been applied to scalar theories [12, 13] and to the colour-electric sector $\sim g T$ of gauge theories in a dimensionally reduced setting [14] as well as in four dimensions [15]. There, a screening mass is generated in ordinary perturbation theory and the resummation of its corrections is merely used as a means to improve the convergence. By contrast, the magnetic mass $\sim g^{2} T$ is entirely non-perturbative. It has to be generated by an infinite resummation and evaluated self-consistently by a gap equation. Since the gauge coupling drops out of the effective expansion parameter, there is no parameter to tune and hence no regime where the resummation is guaranteed to work. Its convergence properties can be judged only after explicit calculations.

In this paper we present a systematic derivation of a gauge invariant resummation scheme using auxiliary fields based on the non-linear sigma model. In particular, we discuss the BRS invariance of the resummation scheme which ensures that the symmetries of the theory are maintained throughout a modified perturbative treatment. We compute the gap equation for 
the magnetic screening mass through two loops in general $R_{\xi}$ gauges and duly find it to be gauge parameter independent, thus correcting an error in an earlier two-loop investigation [16]. The two-loop result amounts to a $\sim 15 \%$ correction to the leading one-loop result, thus pointing at a reasonable convergence of the resummation scheme.

\section{Resummation based on a non-linear sigma model}

Let us now focus on Euclidean Yang-Mills theory,

$$
\mathcal{L}(A)=-\frac{1}{2 g^{2}} \operatorname{Tr} F_{\mu \nu}^{2}
$$

where we use a matrix notation,

$$
F_{\mu \nu}=\left[D_{\mu}, D_{\nu}\right], \quad D_{\mu}=\mathbb{1} \partial_{\mu}-A_{\mu}(x), \quad A_{\mu}(x)=i g T^{a} A_{\mu}^{a}(x),
$$

with hermitean $\mathrm{SU}(N)$ generators $T^{a}, a=1 \ldots N^{2}-1$. We are interested in three dimensions, $\mu, \nu=1 \ldots 3$, where the coupling constant $g^{2}$ carries dimension of mass and is to be identified with $g^{2}=g_{4 \mathrm{~d}}^{2} T$, if the action is viewed as the magnetic part of hot Yang-Mills theory.

The general idea of a resummation is to sum up higher order contributions (infinitely many in our case) into a given order of a perturbative expansion. In order to avoid double counting, these contributions must then be left out at the higher order where they naturally occur, such that the perturbative scheme gets reorganised in some systematic way. (For a discussion of various schemes used in the context of thermal field theory, see [17]). This can be formalised by rewriting the Lagrangian serving for the perturbative expansion as [10]

$$
\mathcal{L}_{\text {eff }}=\frac{1}{\ell}[\mathcal{L}(\sqrt{\ell} X)+\Delta \mathcal{L}(\sqrt{\ell} X)-\ell \Delta \mathcal{L}(\sqrt{\ell} X)]
$$

where $X$ generically stands for the fields. The modification $\Delta \mathcal{L}$ contains fields of the original Lagrangian and possibly auxiliary fields. In particular, if $\Delta \mathcal{L}$ is chosen to represent a mass term for the gluon, this will regulate the infrared divergences. The counting parameter $\ell$ in which one expands is to be set to $\ell=1$ at the end of a calculation, for which the Lagrangian is identical to the original one. However, in a perturbative evaluation to finite order the results will differ from the unresummed ones, the original theory being recovered exactly only at asymptotically high orders. Whether low order calculations are a good approximation to the full answer has to be judged from the apparent convergence of the series and may depend on the observable, just as in ordinary perturbation theory.

A valid resummation scheme has to maintain the symmetries of our gauge theory at every order, for general $\ell$. Clearly, this leaves many conceivable choices for $\Delta \mathcal{L}$, several of which have been tried at one-loop level in the literature [8, 9, 10, 11]. An optimal choice would be based on convergence properties in higher orders. Here we work with a gauged non-linear sigma model, coupling a field $\Phi \in \mathrm{U}(N)$ as

$$
\Delta \mathcal{L}(A, \pi)=\frac{m^{2}}{g^{2}} \operatorname{Tr}\left[\left(D_{\mu} \Phi\right)^{\dagger} D_{\mu} \Phi\right], \quad \Phi^{\dagger} \Phi=\mathbb{1}, \quad \Phi(x)=e^{\pi(x)}, \quad \pi(x)=i \frac{g}{m} T^{a} \pi^{a}(x),
$$


where the $\pi^{a}(x) \in \mathbb{R}$ are auxiliary would-be Goldstone boson field 1 with the same mass dimension as the gauge fields $A_{\mu}^{a}(x)$. Under gauge transformations, $\Phi^{\prime}=U \Phi$ and $\left(D_{\mu} \Phi\right)^{\prime}=$ $U\left(D_{\mu} \Phi\right)$, with the unitary matrix $U=e^{\Lambda(x)}$ and corresponding real coefficients $\Lambda^{a}(x)$, with $\Lambda(x)=i T^{a} \Lambda^{a}(x)$. Thus, $\Delta \mathcal{L}$ provides a mass term for the gauge fields at tree-level while maintaining gauge invariance.

\section{Gauge fixing and BRS-invariance}

In order to do perturbative calculations, a gauge needs to be fixed. It is well known that in Higgs and sigma models the standard covariant gauges lead to non-diagonal or mixing terms in scalar and gauge fields, $\sim\left(\partial_{\mu} \pi\right) A_{\mu}$. This can be avoided by choosing $R_{\xi}$-gauges. In the case of a resummed calculation, however, additional choices have to be made.

We can either take the point of view that our starting point is the resummed theory before gauge fixing as in Eq. (3), and then add gauge fixing and ghost terms to that expression,

$$
\begin{gathered}
\mathcal{L}_{\text {eff }, \mathrm{A}}=\frac{1}{\ell}\{\mathcal{L}(\sqrt{\ell} A)+(1-\ell)[\Delta \mathcal{L}(\sqrt{\ell} A, \sqrt{\ell} \pi) \\
\left.\left.+\mathcal{L}_{\mathrm{gf}, \mathrm{A}}(\sqrt{\ell} A, \sqrt{\ell} \pi)+\mathcal{L}_{\mathrm{FP}, \mathrm{A}}(\sqrt{\ell} A, \sqrt{\ell} \pi, \sqrt{\ell} c)\right]\right\}, \\
\mathcal{L}_{\mathrm{gf}, \mathrm{A}}(A, \pi)=-\frac{1}{g^{2} \xi} \operatorname{Tr}\left\{\left(\left(\partial_{\mu} A_{\mu}\right)-\xi m^{2} \operatorname{Tr}\left(\left(\Phi-\Phi^{\dagger}\right) T^{a}\right) T^{a}\right)^{2}\right\}, \\
\mathcal{L}_{\mathrm{FP}, \mathrm{A}}(A, \pi, c)=\frac{1}{g^{2}} \operatorname{Tr}\left\{2\left(\partial_{\mu} \bar{c}\right)\left(\left(\partial_{\mu} c\right)-\left[A_{\mu}, c\right]\right)+\xi m^{2} \bar{c}\left(\Phi^{\dagger} c+c \Phi\right)\right\},
\end{gathered}
$$

with ghost fields $c=i g T^{a} c^{a}$ and anti-ghost fields $\bar{c}=-i g T^{a} \bar{c}^{a}$. We refer to this gauge fixing procedure as A. For $\ell=1$ we obtain Yang-Mills theory without gauge fixing, as in Eq. (3). This invokes the following Feynman rules for the counter term two-point vertices,

$$
\begin{aligned}
& \text { mom : } \quad \Gamma_{\mu \nu, \mathrm{A}}^{a b}\left(A^{2}\right)=\left(\frac{p_{\mu} p_{\nu}}{\xi}+m^{2} \delta_{\mu \nu}\right) \ell \delta^{a b}, \\
& \longrightarrow \quad: \quad \Gamma_{\mathrm{A}}^{a b}\left(\pi^{2}\right)=\left(p^{2}+\xi m^{2}\right) \ell \delta^{a b}, \\
& \leadsto \bullet \quad: \quad \Gamma_{\mathrm{A}}^{a b}\left(c^{2}\right)=\left(p^{2}+\xi m^{2}\right) \ell \delta^{a b} .
\end{aligned}
$$

Alternatively, we may consider Yang-Mills theory in a covariant gauge and then resum the gauge fixed theory, a strategy which we refer to as procedure B,

$$
\begin{gathered}
\mathcal{L}_{\text {eff, } \mathrm{B}=} \frac{1}{\ell}\{\mathcal{L}(\sqrt{\ell} A)+(1-\ell) \Delta \mathcal{L}(\sqrt{\ell} A, \sqrt{\ell} \pi) \\
\left.\quad+\mathcal{L}_{\mathrm{gf}, \mathrm{B}}(\sqrt{\ell} A, \sqrt{\ell} \pi)+\mathcal{L}_{\mathrm{FP}, \mathrm{B}}(\sqrt{\ell} A, \sqrt{\ell} \pi, \sqrt{\ell} c)\right\}, \\
\mathcal{L}_{\mathrm{gf}, \mathrm{B}}(A, \pi)=-\frac{1}{g^{2} \xi} \operatorname{Tr}\left\{\left(\left(\partial_{\mu} A_{\mu}\right)-(1-\ell) \xi m^{2} \operatorname{Tr}\left(\left(\Phi-\Phi^{\dagger}\right) T^{a}\right) T^{a}\right)^{2}\right\}, \\
\mathcal{L}_{\mathrm{FP}, \mathrm{B}}(A, \pi, c)=\frac{1}{g^{2}} \operatorname{Tr}\left\{2\left(\partial_{\mu} \bar{c}\right)\left(\left(\partial_{\mu} c\right)-\left[A_{\mu}, c\right]\right)+(1-\ell) \xi m^{2} \bar{c}\left(\Phi^{\dagger} c+c \Phi\right)\right\} .
\end{gathered}
$$

\footnotetext{
${ }^{1}$ Note that it is the $\pi$ and not the $\Phi$ who get rescaled as $\pi \rightarrow \sqrt{\ell} \pi$ for the purpose of resummation, Eq. (3).
} 
The corresponding counter term two-point vertices read

$$
\begin{array}{lll}
\text { ㄴom }: & \Gamma_{\mu \nu, \mathrm{B}}^{a b}\left(A^{2}\right)=\left(m^{2} \delta_{\mu \nu}\right) \ell \delta^{a b}, \\
\longrightarrow & : & \Gamma_{\mathrm{B}}^{a b}\left(\pi^{2}\right)=\left(p^{2}+\xi(2-\ell) m^{2}\right) \ell \delta^{a b}, \\
\sim & \Gamma_{\mathrm{B}}^{a b}\left(c^{2}\right)=\left(\xi m^{2}\right) \ell \delta^{a b} .
\end{array}
$$

The two formulations feature non-trivial differences. Note that Eq. (15) contributes to both, order $\sim \ell^{1}$ and $\sim \ell^{2}$. Let us remark here that the gauge fixing and corresponding counter terms used in [8, 16] work only for the gluon pole mass to leading and next-to-leading order, but require generalisation (as above) for higher orders and other observables.

In order to provide gauge invariant results for physical observables order by order in perturbation theory, it is necessary and sufficient that the gauge fixed Lagrangian $\mathcal{L}_{\text {eff }}$ is invariant under BRS-transformations [18, 19]. Note that in the resummed theory with general $\ell$ all fields get rescaled by $\sqrt{\ell}$, and so do the gauge transformations. A BRS-transformation now corresponds to the special choice $\Lambda=\omega \sqrt{\ell} c$. The variations of fields and Faddeev-Popov ghosts under infinitesimal BRS-transformations are

$$
\begin{aligned}
\delta_{\mathrm{B}} A_{\mu}= & \omega\left(\partial_{\mu} c\right)+\omega \sqrt{\ell}\left(c A_{\mu}-A_{\mu} c\right), \\
\delta_{\mathrm{B}} \Phi= & \omega \sqrt{\ell} c \Phi \\
\Rightarrow & \delta_{\mathrm{B}} \pi=\omega \sum_{n=0}^{\infty} \frac{B_{n} \ell^{\frac{n}{2}}}{n !} \sum_{j=0}^{n}(-1)^{j}\left(\begin{array}{c}
n \\
j
\end{array}\right) \pi^{n-j} c \pi^{j}+\mathcal{O}\left(\omega^{2}\right) \\
& \approx \omega c-\frac{\omega \sqrt{\ell}}{2}(\pi c-c \pi)+\frac{\omega \ell}{12}(\pi \pi c-2 \pi c \pi+c \pi \pi)+\mathcal{O}\left(\pi^{4}, \omega^{2}\right), \\
\delta_{\mathrm{B}} c= & \omega \sqrt{\ell} c c, \\
\delta_{\mathrm{B}} \bar{c}= & -\frac{\omega}{\xi}\left(\left(\partial_{\mu} A_{\mu}\right)-(1-\ell) \xi m^{2} \operatorname{Tr}\left(\left(e^{\sqrt{\ell} \pi}-e^{-\sqrt{\ell} \pi}\right) T^{a}\right) T^{a} / \sqrt{\ell}\right),
\end{aligned}
$$

where $B_{n}$ are the Bernoulli numbers. The above transformation refers to $\mathcal{L}_{\text {eff,B }}$; for formulation $\mathrm{A}$, the factor $(1-\ell)$ in $\delta_{\mathrm{B}} \bar{c}$ is absent.

As a non-trivial check we have performed our calculations in both ways, obtaining identical gauge invariant results for both setups. We present our results according to setup B, as it is closer to the standard perturbative treatment.

\section{Relation to SU(2) calculations}

Here, we connect our general $\mathrm{SU}(N)$ parametrisation of the scalar field, Eq. (44), to the special case of $\mathrm{SU}(2)$ treated in [8, 16]. Using $\operatorname{Tr}\left(T^{a}\right)=0, \operatorname{Tr}\left(T^{a} T^{b}\right)=\frac{1}{2} \delta^{a b}$ and $\operatorname{Tr} \mathbb{1}=N$, one gets for the product of two generators the standard expression

$$
\begin{aligned}
T^{a} T^{b} & =\frac{\delta^{a b}}{2 N} \mathbb{1}+\operatorname{Tr}\left(\left\{T^{a}, T^{b}\right\} T^{c}\right) T^{c}+\operatorname{Tr}\left(\left[T^{a}, T^{b}\right] T^{c}\right) T^{c} \\
& \equiv \frac{\delta^{a b}}{2 N} \mathbb{1}+\frac{1}{2} d^{a b c} T^{c} \quad+\frac{1}{2} i f^{a b c} T^{c} .
\end{aligned}
$$


Note that for the special case of SU(2), where $T^{a} \sim \sigma^{a}$ and the Pauli matrices anticommute as $\left\{\sigma^{a}, \sigma^{b}\right\}=2 \delta^{a b} \mathbb{1}$, the totally symmetric structure constants vanish, $d^{a b c}=0$. Hence, in $\mathrm{SU}(2)$ the product of two of our scalar fields is diagonal,

$$
\pi \pi=(i g / m)^{2} \pi^{a} \pi^{b} \frac{1}{2}\left(\frac{\delta^{a b}}{N} \mathbb{1}+d^{a b c} T^{c}\right) \stackrel{\mathrm{SU}(2)}{=}(i g / 2 m)^{2} \pi^{a} \pi^{a} \mathbb{1},
$$

and therefore the field $\Phi$ can be expressed as

$$
\begin{aligned}
& \Phi= e^{\pi}=\cos \frac{\pi}{i}+i \sin \frac{\pi}{i} \stackrel{\mathrm{SU}(2)}{=} \sigma \mathbb{1}+i \bar{\pi}^{a} T^{a} \\
& \text { with } \quad \sigma \equiv \cos \frac{g \sqrt{\pi^{a} \pi^{a}}}{2 m} \approx 1+\mathcal{O}\left(\pi^{2}\right), \\
& \text { and } \bar{\pi}^{a} \equiv \frac{2 \pi^{a}}{\sqrt{\pi^{a} \pi^{a}}} \sin \frac{g \sqrt{\pi^{a} \pi^{a}}}{2 m} \approx \frac{g}{m} \pi^{a}+\mathcal{O}\left(\pi^{3}\right) .
\end{aligned}
$$

Hence, for $N=2$ our model can be recast into the form of the model considered in [8]. For general $N$, however, the coefficients on the right-hand side of $\Phi=\frac{\operatorname{Tr} \Phi}{N} \mathbb{1}+2 \operatorname{Tr}\left(\Phi T^{a}\right) T^{a}$ can not be expressed in a closed form in terms of the real fields $\pi^{a}$. The traceless part of $\Phi$ is part of our construction for the gauge fixing term, see Eqs. (6) and (12), which represents a non-trivial generalisation of $R_{\xi}$ gauges to $\mathrm{SU}(N)$.

\section{Pole mass from a gauge invariant gap equation}

Having designed a general gauge invariant resummation scheme for 3d Yang-Mills theory, we now apply it to a calculation of the gluon self-energy. Its transverse and longitudinal parts $\Pi_{\mathrm{T} / \mathrm{L}}$ are defined as

$$
\Pi_{\mu \nu}^{a b}(p) \equiv \delta^{a b}\left\{\left(\delta_{\mu \nu}-\frac{p_{\mu} p_{\nu}}{p^{2}}\right) \Pi_{\mathrm{T}}\left(p^{2}\right)+\frac{p_{\mu} p_{\nu}}{p^{2}} \Pi_{L}\left(p^{2}\right)\right\} .
$$

The self-energy itself generally is a gauge dependent quantity. However, the pole of the transverse part of a gauge boson propagator, $D_{\mathrm{T}}$, is known to be gauge invariant order by order in perturbation theory [20, 21], and we may employ our resummation scheme to evaluate it. (The longitudinal degrees of freedom with a gauge dependent pole correspond to unphysical would-be Goldstone bosons as usual in Higgs-like theories, and can be gauged away in unitary gauge.) Without resummation the pole of the bare transverse propagator is at $p^{2}=0$, whereas in the resummed theory it gets shifted to $p^{2}=-m^{2}$. Identifying $m$ with the pole of the full propagator, we require that the pole stays at $p^{2}=-m^{2}$ to any loop order. Taylor expanding the self-energy about the pole, the transverse propagator reads

$$
D_{\mathrm{T}}=\frac{1}{p^{2}+m^{2}-\Pi_{\mathrm{T}}\left(p^{2}\right)} p^{2}=-\underline{m}^{2}+\delta p^{2} \frac{\frac{1}{1-\Pi_{\mathrm{T}}^{\prime}\left(-m^{2}\right)}}{-\frac{\Pi_{\mathrm{T}}\left(-m^{2}\right)}{1-\Pi_{\mathrm{T}}^{\prime}\left(-m^{2}\right)}+\delta p^{2}+\mathcal{O}\left(\left(\delta p^{2}\right)^{2}\right)},
$$


where $\left.\Pi_{\mathrm{T}}^{\prime}\left(-m^{2}\right) \equiv \partial_{p^{2}} \Pi_{\mathrm{T}}\left(p^{2}\right)\right|_{p^{2}=-m^{2}}$. Near the pole it then corresponds to a massive propagator with residue $Z\left(m^{2}\right)$,

$$
D_{\mathrm{T}} \propto \frac{Z\left(m^{2}\right)}{p^{2}+m^{2}}, \quad Z\left(m^{2}\right)=\frac{1}{1-\Pi_{\mathrm{T}}^{\prime}\left(-m^{2}\right)},
$$

provided the first term in the denominator of Eq. (24) vanishes. This leads to the gap equation

$$
0 \stackrel{!}{=} \frac{\Pi_{\mathrm{T}}\left(-m^{2}\right)}{1-\Pi_{\mathrm{T}}^{\prime}\left(-m^{2}\right)}
$$

Introducing the $\ell$-expansion of the self-energy, $\Pi_{\mathrm{T}}=\sum_{n \geq 1} \ell^{n} \Pi_{\mathrm{T}}^{(n)}\left(p^{2}\right)$, we now expand the gap equation to the desired order in $\ell$ and evaluate it after setting $\ell=1$. Note that, to every order in $\ell$, the gap equation receives different kinds of self-energy contributions contributing to order $\ell^{n}$. In order to list these separately, we introduce $\Pi_{\mathrm{T}}^{(n-k), k}$ to denote the sum of diagrams with $(n-k)$ loops and $k$ counter term insertions,

$$
\Pi_{\mathrm{T}}^{(n)}=\sum_{k=0}^{n} \Pi_{\mathrm{T}}^{(n-k), k} .
$$

The corresponding gap equation combines gauge dependent contributions of different selfenergies $\Pi_{\mathrm{T}}^{(n-k), k}$ into a gauge invariant quantity.

We perform our calculations using dimensional regularisation, working in $d$ dimensions and with generic gauge fixing parameter $\xi$. Details and intermediate results are relegated to the appendix, from which we collect the results in the following sections.

\subsection{One-loop gap equation}

To leading order $\ell^{1}$, the gap equation Eq. (26) is simply

$$
0=\Pi_{\mathrm{T}}^{(1)}\left(-m^{2}\right)=\Pi_{\mathrm{T}}^{(1), 0}\left(-m^{2}\right)+\Pi_{\mathrm{T}}^{(0), 1}\left(-m^{2}\right) .
$$

The five diagrams shown in Fig. 1 have been computed in a general $R_{\xi}$-gauge in [8] and lead to a $\xi$-independent gap equation when evaluated on the pole. From appendix $\mathrm{A}$, we reproduce these results for $\mathrm{SU}(N)$ as

$$
\begin{aligned}
\Pi_{\mathrm{T}}^{(1), 0}\left(-m^{2}\right) & \stackrel{d=3-2 \epsilon}{\approx} \frac{g^{2} N m}{8 \pi}\left(\frac{3}{4}-\frac{63}{16} \ln 3\right)+\mathcal{O}(\epsilon), \\
\Pi_{\mathrm{T}}^{(0), 1}\left(p^{2}\right) & =m^{2} .
\end{aligned}
$$

Solving the quadratic one-loop gap equation Eq. (28) then yields the well-known solutions

$$
m_{1-\text { loop }}=0 \quad \text { or } \quad m_{1-\text { loop }}=\left(\frac{63}{16} \ln 3-\frac{3}{4}\right) \frac{g^{2} N}{8 \pi}=0.142276 g^{2} N \text {. }
$$




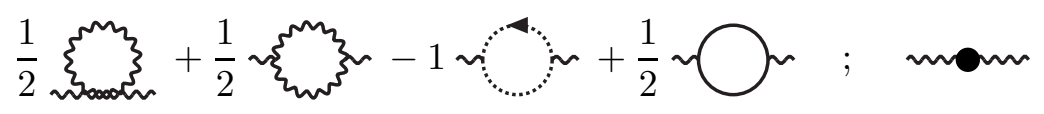

Figure 1: The self-energy diagrams contributing to order $\ell^{1}$. Wiggly/dotted/full lines denote gluons/ghosts/scalars, respectively. In the notation of Eq. (27), the first four diagrams give $\Pi_{\mathrm{T}}^{(1), 0}$, while the last is $\Pi_{\mathrm{T}}^{(0), 1}$.

\section{$5.2 \quad$ Two-loop gap equation}

At order $\ell^{2}$, the gap equation Eq. (26) reads

$$
\begin{aligned}
0 & =\Pi_{\mathrm{T}}^{(1)}\left(-m^{2}\right)\left(1+\left.\partial_{p^{2}} \Pi_{\mathrm{T}}^{(1)}\left(p^{2}\right)\right|_{p^{2}=-m^{2}}\right)+\Pi_{\mathrm{T}}^{(2)}\left(-m^{2}\right) \\
& =\left(\Pi_{\mathrm{T}}^{(0), 1}+\Pi_{\mathrm{T}}^{(1), 0}\right)\left(1+\partial_{p^{2}} \Pi_{\mathrm{T}}^{(1), 0}+\partial_{p^{2}} \Pi_{\mathrm{T}}^{(0), 1}\right)+\Pi_{\mathrm{T}}^{(2), 0}+\Pi_{\mathrm{T}}^{(1), 1} \\
& =m^{2}+\Pi_{\mathrm{T}}^{(1), 0}+\left(\Pi_{\mathrm{T}}^{(1), 1}+m^{2} \partial_{p^{2}} \Pi_{\mathrm{T}}^{(1), 0}\right)+\left(\Pi_{\mathrm{T}}^{(2), 0}+\Pi_{\mathrm{T}}^{(1), 0} \partial_{p^{2}} \Pi_{\mathrm{T}}^{(1), 0}\right),
\end{aligned}
$$

where in the last line we have used Eq. (30) and grouped together terms which will prove to be gauge invariant. There are 38 genuine two-loop diagrams contributing to $\Pi_{\mathrm{T}}^{(2), 0}$, shown in Fig. 2. These can be expressed in terms of six scalar master integrals. Note that in unitary gauge $(\xi \rightarrow \infty$, to be taken before regularisation) the ghosts and pseudo-goldstones decouple leaving only nine diagrams (see also [16]). The one-loop diagrams with one counter term insertion are shown in Fig. 3, A tree-level diagram with two counter term insertions is not one-particle-irreducible and hence does not contribute. From appendix A (where the renormalised $3 \mathrm{~d}$ coupling $g^{2}(\mu)=\mu^{-2 \epsilon} g_{\text {bare }}^{2}$ was introduced), the different contributions to the two-loop gap equation are

$$
\begin{aligned}
& \Pi_{\mathrm{T}}^{(2), 0}+\Pi_{\mathrm{T}}^{(1), 0} \partial_{p^{2}} \Pi_{\mathrm{T}}^{(1), 0} \stackrel{d=3-2 \epsilon}{\approx}\left(\frac{g^{2} N}{8 \pi}\right)^{2}\left(\frac{3}{20 \epsilon}-10.6452+\frac{9}{10}+\frac{3}{10} \ln \frac{\bar{\mu}^{2}}{4 m^{2}}\right)+\mathcal{O}(\epsilon), \\
& \Pi_{\mathrm{T}}^{(1), 1} \stackrel{d=3-2 \epsilon}{\approx} \frac{g^{2} N m}{8 \pi}\left(\frac{21}{8} \ln 3-\frac{9}{2}+\frac{1-4 \xi}{8} \ln \frac{2 \sqrt{\xi}+1}{2 \sqrt{\xi}-1}+\frac{3}{2} \sqrt{\xi}\right)+\mathcal{O}(\epsilon), \\
& \partial_{p^{2}} \Pi_{\mathrm{T}}^{(1), 0} \stackrel{d=3-2 \epsilon}{\approx} \frac{g^{2} N}{8 \pi m}\left(-\frac{21}{32} \ln 3+\frac{33}{8}-\frac{1-4 \xi}{8} \ln \frac{2 \sqrt{\xi}+1}{2 \sqrt{\xi}-1}-\frac{3}{2} \sqrt{\xi}\right)+\mathcal{O}(\epsilon),
\end{aligned}
$$

where all quantities are understood on-shell $\left(p^{2}=-m^{2}\right)$ and for an analytic expression of the two-loop constant 10.6452 we refer to Eq. (62). Note that in general the on-shell self-energy is gauge dependent. However, the parts of the gap equation pertaining to the non-linear sigma model without counter terms, i.e. the first of Eqs. (33), as well as the sum of all counter term contributions are separately gauge invariant, thus leading to a gauge invariant solution for the pole mass in the resummed theory. Our result for the second line of Eq. (33) corrects an error in an earlier calculation for $S U(2)$ [16], which led to a $\xi$-dependent pole. 


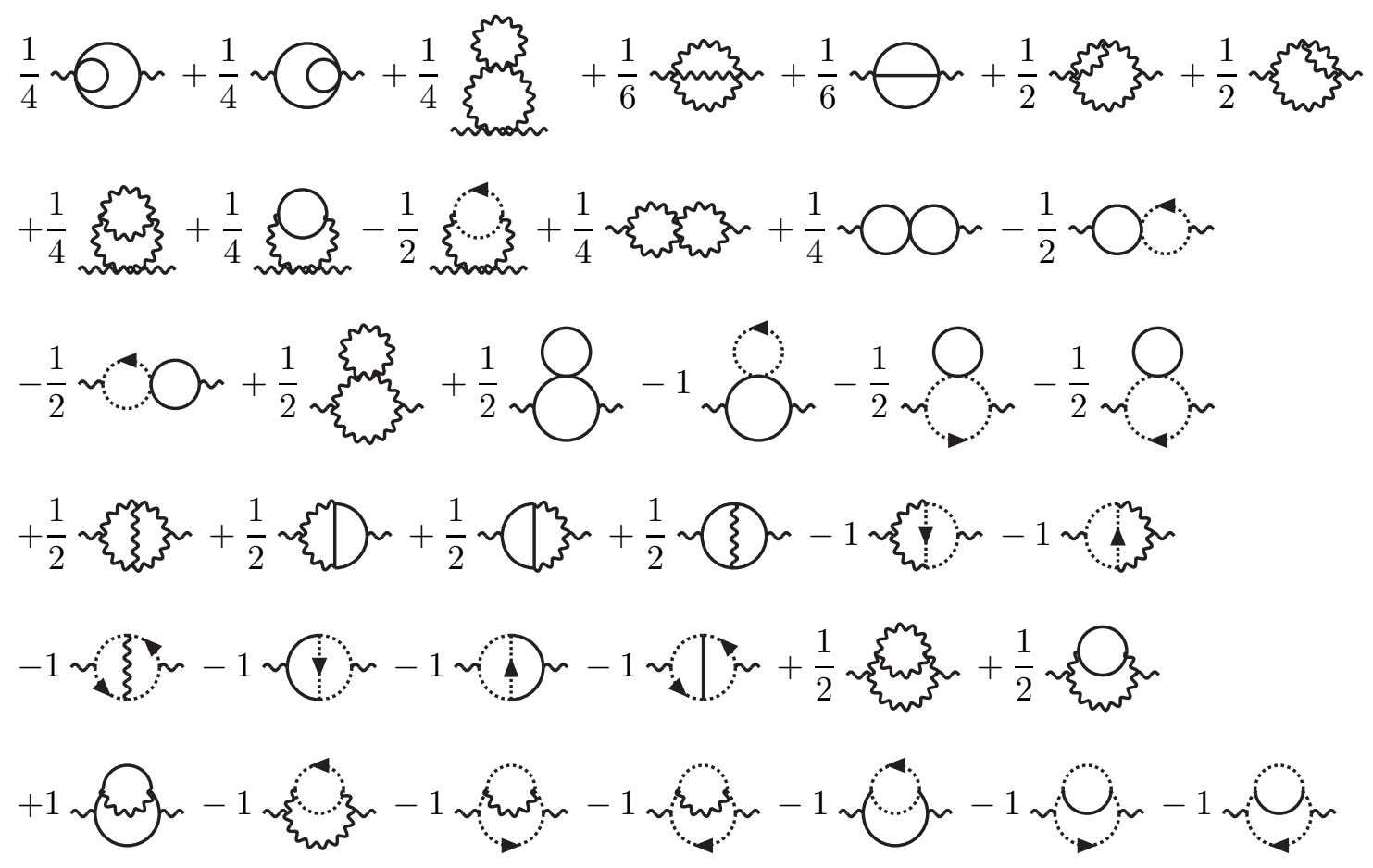

Figure 2: The 38 diagrams contributing to $\Pi_{\mathrm{T}}^{(2), 0}$. Notation as in Fig. 11.

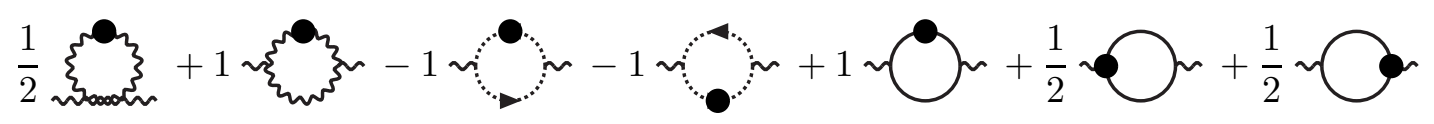

Figure 3: The 7 diagrams contributing to $\Pi_{\mathrm{T}}^{(1), 1}$. Notation as in Fig. 1,

The first of Eqs. (33) features a divergence as $d \rightarrow 3$, which is removed by mass renormalisation according to $m_{\text {bare }}^{2}=m^{2}(\mu)+\delta m^{2}$, where $\delta m^{2}=-\frac{3 x^{2 \epsilon}}{20 \epsilon}\left(\frac{g^{2}(\mu) N}{8 \pi}\right)^{2}$ with $x=1$ $\left(x=4 \pi e^{-\gamma}\right)$ specifying the MS $(\overline{\mathrm{MS}})$ scheme, respectively, and $m^{2}(\mu)$ denotes the renormalised mass. The renormalised two-loop gap equation reads (with $\bar{\mu}^{2}=4 \pi e^{-\gamma} \mu^{2}$ )

$$
0=m^{2}+\frac{g^{2} N m}{8 \pi} \frac{1}{2}\left(\frac{3}{4}-\frac{63}{16} \ln 3\right)+\left(\frac{g^{2} N}{8 \pi}\right)^{2}\left(-10.6452+\frac{9}{10}+\frac{3}{10} \ln \frac{\bar{\mu}^{2}}{4 x m^{2}}\right) .
$$

Note that the renormalisation prescription has introduced scheme as well as scale dependence. In the following we pick the MS scheme $(x=1)$. The scale dependence is formally of higher order: since the bare mass is scale independent, the renormalised mass reacts to a scale variation as $m^{2}(\mu)=m^{2}\left(\mu_{0}\right)-\frac{3}{5}\left(\frac{g^{2} N}{8 \pi}\right)^{2} \ln \left(\mu / \mu_{0}\right)$. In a truncated perturbative series, however, this scale dependence remains and can be taken as an estimate for the size of higher 


\begin{tabular}{|c|c|c|c|c|c|}
\hline$\frac{\bar{\mu}}{g^{2} N}$ & 0.1 & 0.3 & 1 & 3 & 10 \\
\hline$C_{1}$ & 0.1692 & 0.1651 & 0.1605 & 0.1562 & 0.1512 \\
\hline$C_{2}$ & $4.4 \cdot 10^{-9}$ & $1.3 \cdot 10^{-8}$ & $4.4 \cdot 10^{-8}$ & $1.3 \cdot 10^{-7}$ & $4.4 \cdot 10^{-7}$ \\
\hline
\end{tabular}

Table 1: Scale-dependent solutions of the two-loop gap equation Eq. (34), $m=C_{i} g^{2} N$.

order corrections. For a particular choice of scale, $\mu=m$, the logarithm can be absorbed into the pole mass, such that the gap equation reduces to $0=m^{2}-0.07114 g^{2} N m-0.01516 g^{4} N^{2}$, with positive solution $m_{2-\text { loop }}=0.1637 g^{2} N$.

Eq. (34) possesses non-trivial, real solutions which are listed in Table 11 (again in the MS scheme, $x=1$; the second solution, $C_{2}$, is almost always close to zero, and we shall hence still call it trivial). Since the gluon does not correspond to an asymptotic particle state, the scale dependence of its pole mass is expected and in complete analogy to the two-loop pole masses of the electroweak gauge bosons [21]. Note that the pole mass changes by $\lesssim 10 \%$ only as the renormalisation scale is varied over two orders of magnitude. Together with the fact that the two-loop contribution constitutes a $\sim 15 \%$ correction to the leading-order one-loop result, this points to a reasonable convergence of the resummation scheme.

\section{Conclusions}

We have generalised a non-perturbative resummation scheme for three-dimensional YangMills theory based on the non-linear sigma model [8] to $\mathrm{SU}(N)$. Adding and subtracting a covariantly coupled scalar field allows for a gauge invariant gluon mass term regulating infrared divergences encountered in bare perturbation theory. We have established that this leads to gauge invariant physical results by analysing the BRS-invariance of the resummed theory. As an application, we have calculated the transverse gluon propagator and evaluated its pole mass by means of a gap equation, which we explicitly verified to be gauge invariant through two loops, thus correcting an error in [16]. The pole mass requires normalisation. We have employed the minimal subtraction (MS) scheme, through which it acquires a weak scale dependence. We find the two-loop correction to be $\sim 15 \%$ of the leading one-loop result, and the scale dependence $\sim 10 \%$ of the two-loop result when the renormalisation scale is varied over two orders of magnitude. Together, these two features might be indicative of a reasonable convergence behaviour of our resummation scheme.

As a further application, the scheme lends itself to an evaluation of the $g^{6}$-contribution to the thermodynamic pressure in four-dimensional gauge theories. Preliminary investigations up to two loops have been reported in [22], a three-loop calculation is currently under way. 


\section{Acknowledgements}

D.B. and O.P. are supported by the German Bundesministerium für Bildung und Forschung (BMBF), grant no. 06MS9150. Y.S. is supported by the Heisenberg program of the Deutsche Forschungsgemeinschaft (DFG), contract no. SCHR 993/1.

\section{A Details of the calculation}

Due to the somewhat non-standard action with potentially high-order vertices, we automatically generate Feynman rules as well as a model file directly from Eq. (111). For two-loop self-energies, we potentially need vertices with up to six legs.

For notational simplicity, let us write the loop expansion of the bare on-shell transverse self-energy as defined in Eq. (23) as well as its derivatives as

$$
\left.\partial_{p^{2}}^{a} \partial_{m^{2}}^{b} \Pi_{\mathrm{T}}^{\mathrm{bare}}\left(p^{2}\right)\right|_{p^{2}=-m^{2}}=\left(m^{2}\right)^{1-a-b} \sum_{n \geq 1}\left[\frac{g_{\mathrm{bare}}^{2} N J(d, m)}{m^{2}(1-d)}\right]^{n} \hat{\Pi}_{a b}^{(n)}
$$

where the $\hat{\Pi}^{(n)}$ are dimensionless functions of $d, \xi$ and $N$ only which are computed from $n$-loop Feynman diagrams, and $J(d, m)$ is the massive one-loop tadpole integral defined by Eq. (64). Note that the square bracket in dimensional regularisation expands as

$$
\left[\frac{g_{\text {bare }}^{2} N J(d, m)}{m^{2}(1-d)}\right] \stackrel{d=3-2 \epsilon}{\approx} \frac{g^{2} N}{8 \pi m}\left(\frac{\bar{\mu}}{2 m}\right)^{2 \epsilon}\left(1+3 \epsilon+\mathcal{O}\left(\epsilon^{2}\right)\right)
$$

with renormalised coupling (note that $Z_{g^{2}}=1$ in $3 \mathrm{~d}$ ) $g^{2}=\mu^{-2 \epsilon} g_{\text {bare }}^{2}$ and the usual $\overline{\mathrm{MS}}$ scale $\bar{\mu}^{2}=4 \pi e^{-\gamma} \mu^{2}$. From Eq. (35) we explicitly have, in the notation of the main text 2 ,

$$
\begin{aligned}
\Pi_{\mathrm{T}}^{(1), 0}\left(-m^{2}\right) & =m^{2}\left[\frac{g_{\mathrm{bare}}^{2} N J(d, m)}{m^{2}(1-d)}\right] \hat{\Pi}_{00}^{(1)}, \\
\Pi_{\mathrm{T}}^{(2), 0}\left(-m^{2}\right) & =m^{2}\left[\frac{g_{\mathrm{bare}}^{2} N J(d, m)}{m^{2}(1-d)}\right]^{2} \hat{\Pi}_{00}^{(2)}, \\
\left.\partial_{p^{2}} \Pi_{\mathrm{T}}^{(1), 0}\left(p^{2}\right)\right|_{p^{2}=-m^{2}} & =\left[\frac{g_{\mathrm{bare}}^{2} N J(d, m)}{m^{2}(1-d)}\right] \hat{\Pi}_{10}^{(1)}, \\
\Pi_{\mathrm{T}}^{(1), 1}\left(-m^{2}\right) & =-m^{2}\left[\frac{g_{\mathrm{bare}}^{2} N J(d, m)}{m^{2}(1-d)}\right] \hat{\Pi}_{01}^{(1)} .
\end{aligned}
$$

From here, the calculation proceeds via standard methods. All diagrams that we need are generated with QGRAF [23]. We then shift momenta to our conventions, apply colour and Lorentz projectors, perform colour traces via the Fierz-identity, rewrite scalar products in terms of inverse propagators, and perform derivatives for $\hat{\Pi}_{a b}$ on the integrand level. Using

\footnotetext{
${ }^{2}$ The last of the four relations is non-trivial and follows from realising the mass-shift $m^{2} \rightarrow(1-\ell) m^{2}$ needed for the resummed theory Eq. (11) by a translation operator $\exp \left(p^{2} \ell \partial_{m^{2}}\right)$ followed by the on-shell condition.
} 
finally the on-shell condition $p^{2}=-m^{2}$, we obtain an intermediate result for the $\hat{\Pi}$ in terms of dimensionless one- and two-loop on-shell integrals $\hat{I}$, defined as

$$
\begin{aligned}
\hat{I}\left(s_{1}, \ldots, s_{4}\right) \equiv & \left.\frac{1}{J(d, 1)} \int \frac{\mathrm{d}^{d} k}{(2 \pi)^{d}} \frac{1}{\left[k^{2}+s_{3}\right]^{s_{1}}} \frac{1}{\left[(k-p)^{2}+s_{4}\right]^{s_{2}}}\right|_{p^{2}=-1} \\
\hat{I}\left(s_{1}, \ldots, s_{10}\right) \equiv & \frac{1}{[J(d, 1)]^{2}} \int \frac{\mathrm{d}^{d} k_{1}}{(2 \pi)^{d}} \int \frac{\mathrm{d}^{d} k_{2}}{(2 \pi)^{d}} \frac{1}{\left[k_{1}^{2}+s_{6}\right]^{s_{1}}} \frac{1}{\left[k_{2}^{2}+s_{7}\right]^{s_{2}}} \times \\
& \times\left.\frac{1}{\left[\left(k_{1}-k_{2}\right)^{2}+s_{8}\right]^{s_{3}}} \frac{1}{\left[\left(k_{1}-p\right)^{2}+s_{9}\right]^{s_{4}}} \frac{1}{\left[\left(k_{2}-p\right)^{2}+s_{10}\right]^{s_{5}}}\right|_{p^{2}=-1},
\end{aligned}
$$

where the normalisation factor $J$ is a one-loop massive tadpole as defined in Eq. (64).

In a next step, using symmetries and reduction relations following from systematic use [24, 25] of integration-by-parts (IBP) identities [26], we arrive at $d$-dimensional expressions in terms of a few master integrals, as listed below.

\section{A.1 One-loop computations}

Applying the necessary projectors on the sum of diagrams depicted in Fig. 1 as well as its $p^{2}$-derivative (which we take at the integrand level) and performing a reduction to master integrals, we obtain the $d$-dimensional results

$$
\begin{aligned}
& \hat{\Pi}_{00}^{(1)}=a_{1} K_{1}+a_{2} K_{2}, \\
& \hat{\Pi}_{10}^{(1)}=b_{1} K_{1}+b_{2} K_{2}+b_{3} K_{1}^{\prime}+b_{4} K_{2}^{\prime},
\end{aligned}
$$

with master integrals $K_{i}$ listed in App. B and coefficients

$$
\begin{aligned}
a_{1} & =\frac{9}{8}(4 d-5), & a_{2} & =\frac{1}{4}(2 d-3)(2 d-5), \\
b_{1} & =\frac{3}{16}(d-2)(4 d-5), & b_{2} & =\frac{1}{8}\left(12 d^{2}-31 d+18\right), \\
b_{3} & =\frac{1}{4}(1-4 \xi), & b_{4} & =\frac{1}{2}(3-2 d) .
\end{aligned}
$$

Similarly, either from the diagrams of Fig. 3 and using Eq. (40) in reverse, or directly from the $m^{2}$-derivative (easily taken at the integrand level) of the first four diagrams of Fig. 1,

$$
\begin{aligned}
\hat{\Pi}_{01}^{(1)} & =4 b_{1} K_{1}+b_{5} K_{2}+b_{3} K_{1}^{\prime}+b_{4} K_{2}^{\prime}, \\
b_{5} & =\frac{1}{2}\left(d^{3}-3 d^{2}+4 d-3\right) .
\end{aligned}
$$

Note that $\hat{\Pi}_{00}^{(1)}$ is gauge parameter independent, while $\hat{\Pi}_{10}^{(1)}$ and $\hat{\Pi}_{01}^{(1)}$ are not. However, their difference $\hat{\Pi}_{10}^{(1)}-\hat{\Pi}_{01}^{(1)}=-\frac{d-2}{2} \hat{\Pi}_{00}^{(1)}$ is gauge invariant. Let us remark that this relation between the three $\hat{\Pi}^{(1)}$ is not just a coincidence, but the one-loop on-shell case of a general relation which follows from using that dimensional analysis gives $\Pi_{\mathrm{T}} \sim$ mass $^{2}$ and $g^{2} \sim$ mass $^{4-d}$, such that

$$
\left(p^{2} \partial_{p^{2}}+m^{2} \partial_{m^{2}}+\frac{4-d}{2} g^{2} \partial_{g^{2}}\right) \Pi_{\mathrm{T}}\left(p^{2}, m^{2}, g^{2}\right)=1 \cdot \Pi_{\mathrm{T}}\left(p^{2}, m^{2}, g^{2}\right) \text {. }
$$




\section{A.2 Two-loop computations}

Applying the necessary projectors on the sum of diagrams depicted in Fig. 2 and performing a reduction to master integrals, we obtain the $d$-dimensional result

$$
\begin{aligned}
\hat{\Pi}_{00}^{(2)}= & c_{1} K_{3}+c_{2} K_{4}+c_{3} K_{5}+c_{4} K_{6}+c_{5} K_{1} K_{1}+c_{6} K_{1} K_{2}+c_{7} K_{2} K_{2} \\
& -a_{1} b_{3} K_{1} K_{1}^{\prime}-a_{2} b_{3} K_{2} K_{1}^{\prime}-a_{1} b_{4} K_{1} K_{2}^{\prime}-a_{2} b_{4} K_{2} K_{2}^{\prime},
\end{aligned}
$$

with master integrals $K_{i}$ listed in App. B and coefficients

$$
\begin{aligned}
& c_{1}=\frac{3}{64}(d-1)(176 d-245), \\
& c_{2}=-\frac{3}{64}\left(144 d^{3}-712 d^{2}+1241 d-760\right), \\
& c_{3}=-\frac{10800 d^{4}-70632 d^{3}+165227 d^{2}-166654 d+61752}{192(3 d-4)}, \\
& c_{4}=-\frac{3}{64}(d-2)\left(32 d^{3}-312 d^{2}+656 d-405\right), \\
& c_{5}=\frac{3}{128}\left(32 d^{2}-148 d+155\right), \\
& c_{6}=-\frac{3}{16}\left(16 d^{4}-188 d^{3}+668 d^{2}-940 d+465\right), \\
& c_{7}=-\frac{1}{32} \frac{2 d-3}{3 d-4}\left(24 d^{5}-164 d^{4}+452 d^{3}-680 d^{2}+597 d-242\right) .
\end{aligned}
$$

Individual diagrams do have contributions to $\hat{\Pi}_{00}^{(2)}$ that are proportional to $1 / N^{4}$ or $1 / N^{2}$, but these cancel in the sum, leaving $\hat{\Pi}_{00}^{(2)} N$-independent.

Note that $\hat{\Pi}_{00}^{(2)}+\hat{\Pi}_{00}^{(1)} \hat{\Pi}_{10}^{(1)}$ as well as $\hat{\Pi}_{00}^{(2)}+\hat{\Pi}_{00}^{(1)} \hat{\Pi}_{01}^{(1)}$ are gauge parameter independent.

\section{A.3 Results in 3d}

Let us here collect the expansions around $d=3-2 \epsilon$ of Eqs. (43), (44) and (48)

$$
\begin{array}{lll}
\hat{\Pi}_{00}^{(1)} & \stackrel{d=3-2 \epsilon}{\approx} & -\frac{63}{16} \ln 3+\frac{3}{4}+\mathcal{O}(\epsilon) \approx-3.57579+\mathcal{O}(\epsilon) \\
\hat{\Pi}_{10}^{(1)} & \stackrel{d=3-2 \epsilon}{\approx} & -\frac{21}{32} \ln 3+\frac{33}{8}-\frac{1-4 \xi}{8} \ln \frac{2 \sqrt{\xi}+1}{2 \sqrt{\xi}-1}-\frac{3}{2} \sqrt{\xi}+\mathcal{O}(\epsilon) \\
\hat{\Pi}_{01}^{(1)} & \stackrel{d=3-2 \epsilon}{\approx} & -\frac{21}{8} \ln 3+\frac{9}{2}-\frac{1-4 \xi}{8} \ln \frac{2 \sqrt{\xi}+1}{2 \sqrt{\xi}-1}-\frac{3}{2} \sqrt{\xi}+\mathcal{O}(\epsilon)
\end{array}
$$

satisfying $\hat{\Pi}_{10}^{(1)}-\hat{\Pi}_{01}^{(1)} \stackrel{d=3-2 \epsilon}{\approx}-\frac{1}{2} \hat{\Pi}_{00}^{(1)}+\mathcal{O}(\epsilon)$, as well as of Eq. (51) (subtracting $\xi$-dependence)

$$
\begin{aligned}
\hat{\Pi}_{00}^{(2)}+\hat{\Pi}_{00}^{(1)} \hat{\Pi}_{10}^{(1)} \stackrel{d=3-2 \epsilon}{\approx} & \frac{3}{20 \epsilon}+\frac{849}{32} \frac{f(1 / 3)-f(7 / 9)}{\sqrt{2}}-\frac{1329}{512}\left(-\frac{\pi^{2}}{6}+6 \operatorname{Li}_{2}(1 / 3)-2 \operatorname{Li}_{2}(1 / 9)\right) \\
& +\frac{17069}{4800}+\frac{16761}{320} \ln 2-\frac{369}{8} \ln 3-\frac{9}{512} \ln ^{2} 3+\mathcal{O}(\epsilon) \\
\approx & \frac{3}{20 \epsilon}-10.6452+\mathcal{O}(\epsilon) .
\end{aligned}
$$


For comparison, $\hat{\Pi}_{10}^{(1)}=4 \pi f_{2}(\xi)+\mathcal{O}(\epsilon)$ in the notation of Eq. (21) in [16], but $\hat{\Pi}_{01}^{(1)} \neq$ $-4 \pi f_{1}(\xi)+\mathcal{O}(\epsilon)$, pointing to an error in that reference.

\section{B Master integrals}

We like to work with dimensionless and measure independent integrals, so let us normalise each loop by the massive one-loop tadpole integral $J(d, m)$, which for our choice of measure reads

$$
\begin{aligned}
& J(d, m) \equiv J \equiv \quad \equiv \quad \frac{\mathrm{d}^{d} k}{(2 \pi)^{d}} \frac{1}{k^{2}+m^{2}}=\frac{1}{m^{2}}\left(\frac{m^{2}}{4 \pi}\right)^{d / 2} \Gamma(1-d / 2) \\
& d={\underset{3}{2}}^{2} 2 \epsilon-\frac{m}{4 \pi}\left(\frac{\pi e^{-\gamma}}{m^{2}}\right)^{\epsilon}\left(1+2 \epsilon+\mathcal{O}\left(\epsilon^{2}\right)\right) .
\end{aligned}
$$

From reference [27] (and using Eq. (1) therein as well as Eqs. (1,3,9-11,14) of [28] for $K_{3}$ ), we get expansions around $d=3-2 \epsilon$ for all on-shell master integrals that we need:

$$
\begin{aligned}
& K_{1}=\left[m^{2}-\bigcirc-/ J\right]_{\mathrm{os}}=\hat{I}(1,1,1,1) \\
& \stackrel{d=3-2 \epsilon}{\approx}-\frac{\ln 3}{2}+\mathcal{O}(\epsilon), \\
& K_{1}^{\prime}=\left[m^{2}-\left(\begin{array}{c}
\xi m^{2} \\
\xi m^{2}
\end{array}-/ J\right]_{\mathrm{os}}=\hat{I}(1,1, \xi, \xi)\right. \\
& \stackrel{d=3-2 \epsilon}{\approx}-\frac{1}{2} \ln \frac{2 \sqrt{\xi}+1}{2 \sqrt{\xi}-1}+\mathcal{O}(\epsilon) \\
& K_{1}^{\prime \prime}=\left[m^{2}-\left\{m^{2}-/ J\right]_{\mathrm{os}}=\hat{I}(1,1,1, \xi)\right. \\
& \stackrel{d=3-2 \epsilon}{\approx}-\frac{1}{2} \ln \frac{\sqrt{\xi}+2}{\sqrt{\xi}}+\mathcal{O}(\epsilon), \\
& K_{3}=\left[m^{6}-\bigcirc-/ J^{2}\right]_{\mathrm{os}}=\hat{I}(1,1,1,1,1,1,1,1,1,1) \\
& \stackrel{d=3-2 \epsilon}{\approx} \frac{f(1 / 3)-f(7 / 9)}{\sqrt{2}}+\mathcal{O}(\epsilon) \text { with } \quad f(x) \equiv \Im \operatorname{Li}_{2}\left(x+i \sqrt{1-x^{2}}\right) \\
& \approx 16 \pi^{2} \times 0.000245310499 \cdots+\mathcal{O}(\epsilon) \text {, } \\
& K_{4}=\left[m^{4} \bigodot-/ J^{2}\right]_{\mathrm{os}}=\hat{I}(1,1,1,0,1,1,1,1,0,1) \\
& \stackrel{d=3-2 \epsilon}{\approx} \frac{1}{8}\left[\ln ^{2} 3-\frac{\pi^{2}}{6}+6 \operatorname{Li}_{2}(1 / 3)-2 \operatorname{Li}_{2}(1 / 9)\right]+\mathcal{O}(\epsilon) \\
& \approx 16 \pi^{2} \times 0.00121156 \cdots+\mathcal{O}(\epsilon), \\
& K_{5}=\left[m^{2} \bigcirc / J^{2}\right]_{\mathrm{os}}=\hat{I}(1,0,1,0,1,1,0,1,0,1) \\
& \stackrel{d=3-2 \epsilon}{\approx} \frac{1}{4 \epsilon}+\left(\frac{1}{2}-2 \ln 2\right)+\mathcal{O}(\epsilon) \text {. }
\end{aligned}
$$


Two trivial one-loop massive vacuum master integrals read

$$
\begin{aligned}
& K_{2}=[\bigcirc / J]=\hat{I}(1,0,1,0)=1, \\
& K_{2}^{\prime}=\left[\xi^{2} / J\right]=\hat{I}(1,0, \xi, 0)=\xi^{(d-2) / 2} .
\end{aligned}
$$

One non-trivial fully massive vacuum master integral, expanded around $d=3-2 \epsilon$, reads (cf. reference [29]):

$$
\begin{aligned}
& K_{6}=\left[m^{2} \bigcirc / J^{2}\right]=\hat{I}(1,1,1,0,0,1,1,1,0,0) \\
& \underset{\substack{d=3-2 \epsilon}}{\approx} \frac{1}{4 \epsilon}-\left(\frac{1}{2}+\ln \frac{3}{2}\right)+\mathcal{O}(\epsilon) \approx \frac{1}{4 \epsilon}-0.9054651081+\mathcal{O}(\epsilon) .
\end{aligned}
$$

\section{References}

[1] A. D. Linde, Phys. Lett. B 96 (1980) 289.

[2] D. J. Gross, R. D. Pisarski and L. G. Yaffe, Rev. Mod. Phys. 53 (1981) 43.

[3] A. Cucchieri, D. Dudal, T. Mendes and N. Vandersickel, arXiv:1202.0639 [hep-lat].

[4] O. Philipsen, Nucl. Phys. B 628 (2002) 167 hep-lat/0112047.

[5] D. Karabali and V. P. Nair, Nucl. Phys. B 464 (1996) 135 hep-th/9510157.

[6] V. P. Nair, arXiv:1201.0977 [hep-th].

[7] K.-I. Kondo, arXiv:1202.4162 [hep-th].

[8] W. Buchmüller and O. Philipsen, Nucl. Phys. B 443 (1995) 47 arXiv:hep-ph/9411334; O. Philipsen, hep-ph/9406307.

[9] G. Alexanian and V. P. Nair, Phys. Lett. B 352 (1995) 435 arXiv:hep-ph/9504256.

[10] R. Jackiw and S.-Y. Pi, Phys. Lett. B 403 (1997) 297-303 arXiv:hep-th/9703226.

[11] J. M. Cornwall, Phys. Rev. D 10 (1974) 500.

[12] F. Karsch, A. Patkos and P. Petreczky, Phys. Lett. B 401 (1997) 69 hep-ph/9702376.

[13] J. O. Andersen and L. Kyllingstad, Phys. Rev. D 78 (2008) 076008 arXiv:0805.4478 [hep-ph]].

[14] J. P. Blaizot, E. Iancu and A. Rebhan, Phys. Rev. D 68 (2003) 025011 hep-ph/0303045.

[15] J. O. Andersen, M. Strickland and N. Su, arXiv:0911.0676 [hep-ph]. 
[16] F. Eberlein, Phys. Lett. B 439 (1998) 130 hep-ph/9804460.

[17] J. P. Blaizot, E. Iancu and A. Rebhan, arXiv:hep-ph/0303185.

[18] C. K. Becchi, A. Rouet and R. Stora, Annals Phys. 98 (1976) 287-321.

[19] J. Zinn-Justin, Quantum field theory and critical phenomena, Int. Ser. Monogr. Phys. 113 (2002) 1.

[20] A. K. Rebhan, Phys. Rev. D 48 (1993) 3967 hep-ph/9308232.

[21] F. Jegerlehner, M. Y. Kalmykov and O. Veretin, Nucl. Phys. B 641 (2002) 285 hep-ph/0105304.

[22] O. Philipsen, D. Bieletzki and Y. Schröder, PoS QCD -TNT09 (2009) 052 arXiv:0911.3595 [hep-ph]].

[23] P. Nogueira, J. Comput. Phys. 105 (1993) 279; Nucl. Instrum. Meth. A 559 (2006) 220.

[24] O. V. Tarasov, Nucl. Phys. B 502 (1997) 455 hep-ph/9703319.

[25] S. Laporta, Int. J. Mod. Phys. A 15 (2000) 5087 hep-ph/0102033.

[26] K. G. Chetyrkin and F. V. Tkachov, Nucl. Phys. B 192 (1981) 159; F. V. Tkachov, Phys. Lett. B 100 (1981) 65.

[27] A. K. Rajantie, Nucl. Phys. B 480 (1996) 729 [Erratum-ibid. B 513 (1998) 761] hep-ph/9606216.

[28] D. J. Broadhurst, hep-th/9806174.

[29] Y. Schröder and A. Vuorinen, hep-ph/0311323; K. Kajantie, M. Laine, K. Rummukainen and Y. Schröder, JHEP 0304 (2003) 036 [hep-ph/0304048]. 